\title{
Facultad de Ciencias Médicas, 100 años de vida. Homenaje a un maestro.
}

\author{
Faculty of Medical Sciences, 100 years of life. \\ Tribute to a mentor.
}

\author{
Daniela A. Zicre, Roxana M. Lattante, Ana L. Nocito. \\ Rosario. Rosario; Argentina.

\begin{abstract}
Autor por correspondencia: Ana Lía Nocito - alnocito@gmail.com Conflicto de intereses: no presenta.
\end{abstract}

Cátedra de Anatomía y Fisiología Patológicas. Facultad de Ciencias Médicas. Universidad Nacional

\begin{abstract}
Resumen
La Facultad de Ciencias Médicas de Rosario celebra en 2020 su primer centenario y el objetivo de esta publicación es homenajearla. Revisamos la historia fundacional del Hospital Centenario, la Facultad de Medicina y de nuestra Cátedra de Anatomía Patológica. Exaltamos la figura del primer profesor a cargo Dr. Fernando Ruiz, alma mater de su creación y organización, quien diseñó los Trabajos Especiales, realizados por los alumnos de aquel entonces, bajo su tutela. Revisamos los Trabajos que se conservan, realizados entre 1923 y 1930 por alumnos, en su mayoría estudiantes varones, junto a un puñado de mujeres, pioneras en la Medicina de Rosario. Realizamos un relevamiento de las patologías más frecuentes de aquella época: enfermedades infecciosas, patología cardiovascular y neoplasias, presentadas en verdaderas creaciones artísticas que forman parte del patrimonio histórico de nuestra Facultad. La vida del Dr. Ruiz, extinguida en pleno ejercicio de sus funciones, ha dejado una llama encendida, en sus discípulos y alumnos, que aún perdura en las sucesivas generaciones de patólogos de nuestra querida Facultad.
\end{abstract}

Palabras clave: Historia. Patología. Docencia.

\begin{abstract}
The Rosario School of Medicine celebrates its first centenary in 2020 and the objective of this publication is to honor it. We review the founding history of the Centennial Hospital, the Faculty of Medicine and our Chair of Pathology. We exalt the figure of the first profesor in charge of Dr. Fernando Ruiz, alma mater of its creation and organization, who designed the Special Works, carried out by the students of that time, under his tutelage. We review the Works that are preserved, carried out between 1923 and 1930 by students, mostly students men, along with a handful of pioneering women in Rosario's Medicine. We carry out a survey of the most frequent pathologies of that time: infectious diseases, cardiovascular pathology and neoplasms, presented in true artistic creations that are part of the historical heritage of our Faculty. The life of Dr. Ruiz extinguished in full exercice of his functions, has left a burning flame, in his disciples and students, which still lasts in the successive generations of pathologists of our beloved Faculty.
\end{abstract}

Keywords: History. Pathology. Teaching. 
Transcurre el año 2020 y nuestra querida Facultad de Ciencias Médicas cumple 100 años. Pensando en cómo celebrarlo desde la Cátedra de Anatomía Patológica, pensamos realizar una presentación oral donde exponer cómo se desarrollaba en 1923 el cursado de la materia para alumnos y profesores. La pandemia lo frustró.

Esta historia, en donde coexisten enseñanza, aprendizaje, dedicación, amor y entrega es nuestro humilde homenaje.

\section{Los inicios: hospital Centenario, Facultad de Medicina}

En ocasión de conmemorarse el primer Centenario de la Revolución de Mayo Cornelio Casablanca propuso el 18 de abril de 1910 la construcción del Hospital Centenario y de una Escuela de Enseñanza Médica en Rosario. En su inolvidable discurso manifestó el anhelo que el hospital fuera un monumento perdurable que recordara en tiempos venideros el vibrante civismo de sus hijos $(1,2)$.

El Hospital se construyó con el aporte de la comunidad rosarina, subsidios del estado nacional, provincial y municipal. Este último donó cuatro manzanas en las cuales se edificó el Hospital y la Facultad de Medicina. La piedra fundamental fue colocada el 24 de mayo sobre Avenida Francia y el edificio correspondiente al Hospital del Centenario fue proyectado por el arquitecto René Barbá con la colaboración del Dr. Tomas Varzi. Se construyó en varias etapas y paulatinamente fueron habilitadas salas, pabellones y subsuelos.

Transcurría el año1920 y las dos Facultades de Medicina existentes en nuestro país, Buenos Aires (UBA) y Córdoba (UNC), no satisfacían los requerimientos de la época en cuanto a la formación de profesionales.

Rosario ya contaba con el Hospital Provincial del Centenario, pero todos continuaban soñando con la Escuela de Medicina anexa al Hospital. Año tras año muchos personajes ilustres lucharon para que esto pudiera lograrse: Jorge Raúl Rodríguez, Federico Pinedo, Luis Agote, hasta que un 17 de octubre de 1919 se promulgó la Ley 10861 de creación de la Universidad Nacional del Litoral.

Al igual que el hospital Centenario el edificio fue proyectado por el arquitecto René Barba y el Dr Tomas Varzi. El majestuoso frontispicio triangular fue obra del arquitecto Guillermo Gianninazzi y en él se destacan el monumento a los padres y en la porción inferior dos grifos orientados en dirección este y oeste (3).

Finalmente, el 9 de abril de 1920 fue designado como primer decano el Dr José Benjamín Avalos y las clases dieron comienzo el 1 de junio del mismo año a las 8 de la mañana. La primer clase estuvo a cargo del Profesor Titular de la Cátedra de Histología Normal y Embriología Dr. Tomás Cerrutti (4).

\section{El legado del Profesor Dr Fernando Román Ruiz}

En 1887 el Profesor Titular de la Cátedra de estudios anatomopatológicos de la UBA era el Dr Telémaco Susini (5). Había estudiado en dicha universidad y en Europa fue discípulo de Luis Pasteur y Roberto Koch. Siempre sintió una profunda inclinación hacia todo lo que se relacionara con la anatomía humana, más precisamente por las estructuras y enfermedades que las afectaban, es decir por la ANATOMIA PATOLOGICA. Tuvo discípulos de excelencia: José Badías, Fernando Ruiz, Horacio Piñero, Gregorio Araoz Alfaro, Carlos Malbrán, José Arce, Angel Roffo $(6,7,8)$.

Ante el inminente comienzo de las clases en la Facultad de Medicina de Rosario, le solicitaron al Profesor Susini que nombrara a alguno de sus discípulos para que se desempeñara como profesor en la Cátedra de Patología de dicha Facultad. Así fue como el Dr Fernando Ruiz arribó a Rosario.

\section{Camino trazado por el Profesor Dr. Fernando Román Ruiz}

El Dr Ruiz se hizo cargo de la Cátedra de Patología mediante un contrato registrado el 1 de enero de 1921 y a partir de allí dedicó sus días a preparar las instalaciones, organizar, equipar y buscar colaboradores para iniciar el dictado de la materia.

El Dr Joaquín Llambías, 2do Profesor Titular de la Cátedra de Patología de la UBA y un buen amigo del Dr Ruiz, fue invitado por éste para dictar la primera clase de la materia el 20 de marzo de 1921.

El Profesor Fernando Román Ruiz poseía una sólida formación profesional, tanto en el aspecto docente como en la actividad asistencial. Fue el primer profesor de nuestra Cátedra, a ella le brindó los últimos quince años de su vida, con dedicación de tiempo completo. Era un hombre enérgico, dedicado a la actividad científica y académica, trabajador incansable y entusiasta. Era el primero en llegar y el último en irse. Estaba en todo, desde una autopsia a cualquier actividad que se llevara a 
cabo en la Cátedra y la Facultad. Fue miembro del Consejo Directivo de la Facultad y fue mecenas y presidente del Club universitario. Su figura, robusta, le valió el apodo de Gordo Ruiz, término acuñado con profundo cariño, para una figura querida y respetada, que estimuló a sus estudiantes y discípulos a una formación de excelencia y nunca olvidó a su maestro, el Profesor Susini. Dejó una huella imborrable en todo aquel que lo conoció. El Profesor Juan Pedro Picena, su discípulo, lo recordaba con sentida emoción y no perdía oportunidad para exaltar los valores humanos del Dr Ruiz (9)

Controlaba cada protocolo de autopsia, el informe de biopsias del Hospital Centenario, elaboraba material didáctico, participaba en trabajos prácticos y realizaba trabajos y presentaciones científicas, sin el menor atisbo de cansancio. Supo organizar el Instituto de Patología, en todos sus aspectos y se rodeó de excelentes colaboradores, clínicos a quienes formó como sus prosectores promoviendo la correlación anatomo clínica. Fueron sus discípulos: Dr José Matías Cid, Dr Juan Pedro "Pucho" Picena (con quien forjó una profunda amistad), Dr Luis A. Chiodin, Dr Gaudencio Fontana, Dr Franzini Herrera y Dr Tomás Ocaña, sólo por mencionar a los más próximos. Diseñó los trabajos especiales (TE) como condición para rendir examen de la asignatura e impulsaba a sus estudiantes a realizar una pormenorizada relación entre las alteraciones morfológicas halladas y los signos y síntomas que presentaba el paciente.

Era un personaje entrañable que convocaba en su casa a los estudiantes de cada grupo, para ver las lesiones al microscopio y organizar la tarea encomendada. No sólo los recibía en su casa, muchas veces era su consejero y hasta ofició como garante de las pensiones que alquilaban.

Ante el fallecimiento del Prof. Joaquín Llambías en 1931 se le invitó a presentarse a concurso de Profesor Titular de la Cátedra de Patología de la Facultad de Medicina de la UBA en virtud de sus cualidades para desempeñar ese cargo y función. La honestidad y compromiso del Dr Ruiz se vio reflejada una vez más en la carta que envió al Decano de la Facultad de Medicina de la UNL en donde estableció los motivos por los cuales no se iba a postular a dicho cargo: no voy a postularme no sólo por mi contrato firmado sino porque estimo que la obra que he iniciado acá no ha terminado y resta mucho por hacer y porque tengo el convencimiento que esta facultad no tiene porque desmerecerse ante la de Buenos Aires $(10,11,12)$

La muerte lo sorprendió inesperadamente en la plenitud de su actividad. Sus alumnos solicitaron que sus restos fueran velados en el Aula donde daba sus magistrales clases y lo escoltaron hasta su morada final. $(13,14)$. Murió en la pobreza, como muchos grandes. Su riqueza estaba representada por el amor y respeto que le brindaban sus discípulos y colegas. El Dr Ruiz está presente, la Cátedra lleva su nombre y el busto erigido en su memoria, emplazado en el hall del primer piso de la Facultad, vigila con recelo la actividad docente y asistencial.

\section{Revisión de los trabajos especiales: desandando el camino del pasado}

Hoy en el marco de los 100 años de la Facultad y a modo de homenaje al gran maestro que fue el Dr Ruiz decidimos realizar la revisión de todos los TE que como ya se mencionara, fueron elaborados por los alumnos del $3^{\circ}$ año de la carrera, entre los años 1923 y 1930. Por entonces, entre 68 y 108 alumnos por año, entusiastas y ávidos por adquirir conocimientos, habilidades y experiencia fueron subyugados por la inigualable capacidad docente y fervorosa vocación y dedicación del Dr Ruiz.

Dichos trabajos consistían en la realización y presentación de una autopsia completa que los estudiantes debían entregar al final del cursado como condición sine qua non para poder acceder al examen final de la materia Anatomía y Fisiología Patológicas.

Cada uno de ellos incluía un análisis exhaustivo de la historia clínica, una detallada descripción macro y microscópica de cada órgano y el desarrollo fisiopatológico del caso. Como corolario, se exponía una revisión bibliográfica, acompañada de material fotográfico, dibujos y/o esquemas, referentes a la patología responsable del diagnóstico anatomopatológico final.

Este magnífico archivo disponible en nuestra cátedra, fue rescatado del voraz incendio que asoló el segundo piso de la Facultad de Ciencias Medicina ese fatídico 11 de septiembre de 2008 y gracias al trabajo de cada uno de los integrantes, docentes y no docentes, pudo recuperarse y restaurarse junto a otros importantes documentos que forman parte de un patrimonio histórico, testigo silencioso de la actividad científica.

Se trata de un total de 214 autopsias completas y 7 monografías; todos prolijamente encuadernados, escritos a mano con minuciosa caligrafía y apenas unos pocos, mecanografiados. Cada trabajo estaba a cargo de una comisión integrada por un número variable de alumnos que nunca superaba un máximo de 7 . 
Su revisión permitió develar datos significativos respecto a cuáles eran entonces las patologías más frecuentes, cuáles sus formas de presentación y diseminación así como la prevalencia por género y rango etario.

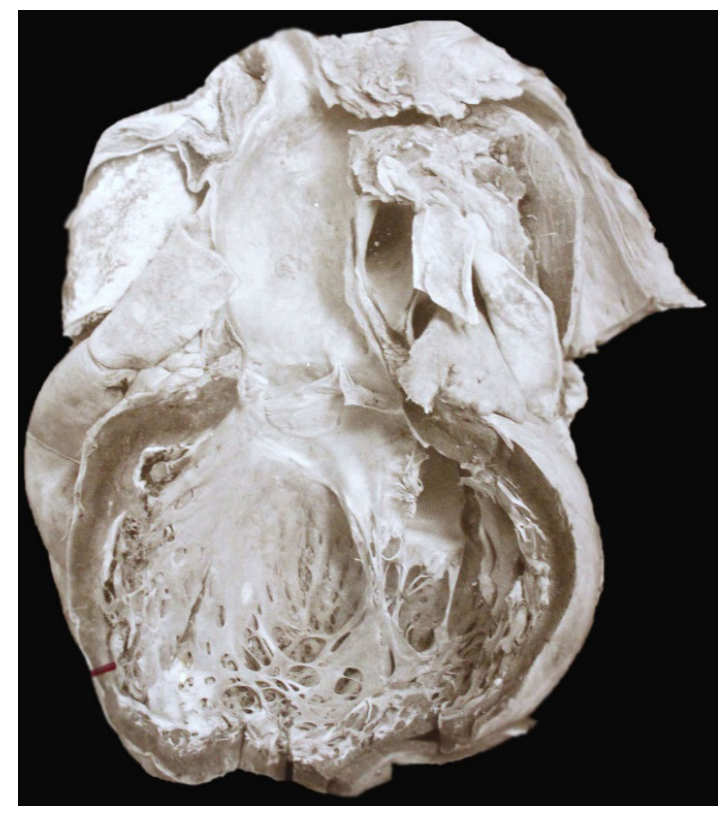

Figura 1: Corazón de buey o cor bovis.

En relación con las autopsias las patologías más frecuentes fueron: tuberculosis, bronconeumonía y las cardiopatías. Esta última diagnosticada en esos años como dilatación cardíaca global, corazón de buey o Corazón de buey o cor bovis (Figura 1) eran atribuídas, según los detalles microscópicos consignados, a causas como miocarditis crónica o intersticial, ateromatosis y/o infartos. También las endocarditis infecciosas y reumática figuraban entre los diagnósticos. Como solía enfatizar otro de los grandes maestros de la patología, el Dr Guillermo Reeves a la luz de los conocimientos actuales los casos diagnosticados como miocarditis, probablemente se debían a etiología chagásica y las afecciones cardíacas muy posiblemente estaban relacionadas a la hipertensión arterial. La tuberculosis (TBC) (39/214) figuraba como la enfermedad infecto-contagiosa preponderante de la época como era de esperar previo a la vacunación con BCG. Se manifestaba en pulmón con patrones macroscópicos variables: patrón neumónico, bronconeumónico, miliar o grandes cavernas y además con formas extensamente diseminadas a órganos como laringe, tráquea, bazo, hígado, ganglios e intestino delgado. Era común la afectación meníngea y de la columna vertebral con secuelas como la fijación de las vértebras y la deformidad de la columna que traía aparejado el Mal de Pott. Otra de las patologías relevantes era la endometritis puerperal post-aborto (13/214) con infartos sépticos en diversos órganos como bazo, riñón y pulmón y el shock séptico como causa final de la muerte. Respecto a enfermedades infecciosas, la sífilis (14/214) ocupaba un lugar destacado con manifestaciones del período terciario como la aortitis sifilítica y gomas en cerebro y pulmón. Fueron contabilizados cinco casos de hidatidosis (Figura 2), tres de fiebre tifoidea y dos de amebiasis.

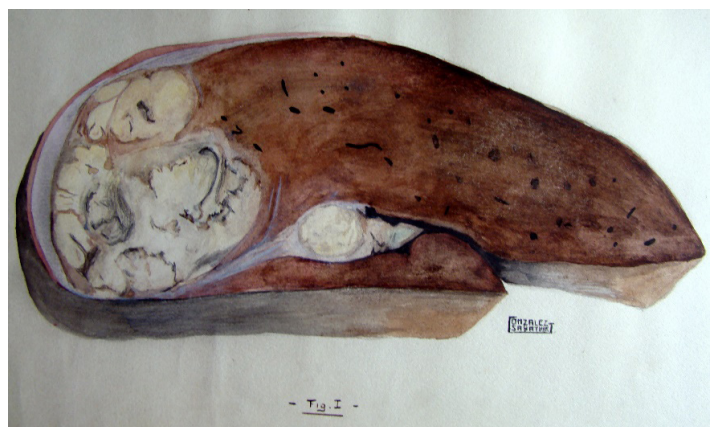

Figura 2: Dibujo de quiste hidatídico en hígado. TE 373/1924 
En cuanto a las neoplasias el adenocarcinoma de estómago encabezó la lista de los tumores malignos (12/214), seguido muy de cerca por el carcinoma de esófago. Algunos tumores figuraban con la nomenclatura de la época como linfosarcoma y sarcoma de pleura (hoy linfomas y mesoteliomas, respectivamente).

Respecto a la prevalencia por género y rango etario, la revisión demostró que las patologías eran notoriamente más frecuentes en hombres y en adultos jóvenes (media de 35 años) con un rango entre 2 y 84 años.

A su vez las monografías consistían en el desarrolllo exhaustivo de un tema, como inflamación o leucosis aguda, con detalles de la fisiopatogenia, clasificación de la época y búsqueda bibliográfica.

Cada uno de los trabajos y monografías se acompañaban con una cuidadosa selección y preparación de las piezas macroscópicas y preparados histológicos para su impresión fotográfica, a lo que se sumaba la ejecución de dibujos, esquemas o gráficos, realizados a mano alzada por los propios alumnos referidos a distintos procesos patológicos.Entre los ejemplos a destacar figura un magnifico esquema del ciclo biológico del Echinococcus granulosus y excelsas fotografías de sífilis pulmonar, TBC esplénica y cor bovis

Cada trabajo era único e irrepetible y todos elaborados con esmero y detalles cuidados. A modo de ejemplo en uno de ellos se podía apreciar una extensa revisión bibliográfica sobre el cáncer en la historia de la medicina con una disquisición bifronte: filosófica y médica. Incluía conceptos de Hipócrates, (quien llamaba karkinoma a los tumores malignos),Virchow y Galeno de Pérgamo, el más grande médico de la antigüedad.

Otros se encontraban precedidos por carátulas que parecían hechas por verdaderos artistas.

Entre ellas hay una que nos hizo reflexionar acerca de su significado. El dibujo fue realizado por el alumno M Hierro y en él se visualiza una calavera, una víbora y una vela. ¿Qué lo llevó a realizar ese dibujo? Conjeturamos una posible relación entre la muerte, inmortalidad o neotenia con la sabiduría o el rejuvenecimiento (serpiente de la vara o báculo de Asclepio/Esculapio) y la iluminación como equivalente de la imaginación, la inteligencia y la intuición ¡Cuanta creatividad! (Figura 3).

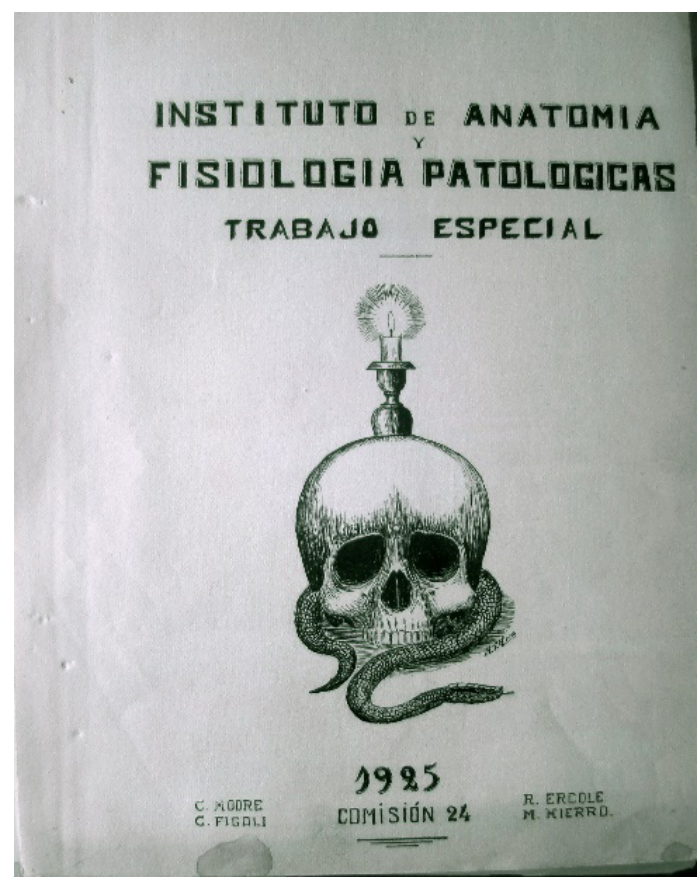

Figura 3: Carátula de comisión 24. Año 1925.

Entre estos trabajos especiales se encontró algunos que pertenecieron a reconocidos médicos rosarinos y entre ellos se puede mencionar, entre otros: Dr J Premoli J, TE 224/1923, Dr Luis Gomez Gonzalez Sabathie TE 373/1924, Dr José María Fernández TE 340/1924, Dr José Celoria TE 805/1926, Dr Germán Rodriguez Roselli TE 940/1927,Dr Rafael Pineda TE 1841/1930.

También fueron hallados los trabajos realizados por las primeras médicas recibidas en nuestra Facultad. La Dra Francisca Montaut de Osuna, TE 410/1924 $(16,17)$ que fue la primera en obtener 
su título en el año 1929. Su trabajo fue presentado en el mes de noviembre de 1924 y se realizó en conjunto con Pablo Pereyra Peralta y Juan Carlos Alvarez. El trabajo fue escrito con una máquina de escribir con cinta azul, revela valiosas descripciones macroscópicas y microscópicas de una necropsia correspondiente a una paciente de 65 años, análisis de las causas mórbidas y concluye con consideraciones finales y la causa de muerte. En la misma se encontró, como lesiones relevantes, hipertrofia cardíaca, leve miocarditis intersticial, arterioesclerosis, leiomioma uterino, esteatosis hepática, arteriolonefroesclerosis y bronquitis catarral.

Si bien todos los trabajos son inéditos y representan verdaderos estudios anatomopatológicos se debe destacar, por la autoría, el realizado por José María Fernandez (18). Médico dermatólogo, leprólogo que enorgullece a Rosario y Argentina, reconocido a nivel mundial fundamentalmente por sus estudios sobre lepra. Recorrió los mayores leprocomios del mundo y efectuó múltiples publicaciones de esta enfermedad entre las que se destaca aquella referida a la reacción cutánea posteriormente conocida por su nombre (19). Trabajó arduamente en el Hospital Carrasco y según su director Hernan Kruse era conmovedor verlo trabajar desde temprano al lado de esos enfermos, una misión natural que no todos estábamos dispuestos a afrontar. En 1940 obtiene por concurso el cargo de Prof Titular de la Cátedra de Dermatología sucediendo a su maestro Enrique Fidanza y entre 1955-57 se desempeñó como rector de la UNL (20).

De él afirmó el Prof. Dr Bernardo Alberto Houssay, *el Dr. Fernández no solamente tiene ideas originales para la investigación científica, sino que además ha sabido guiar a muchos médicos en distintos campos de investigación original*.

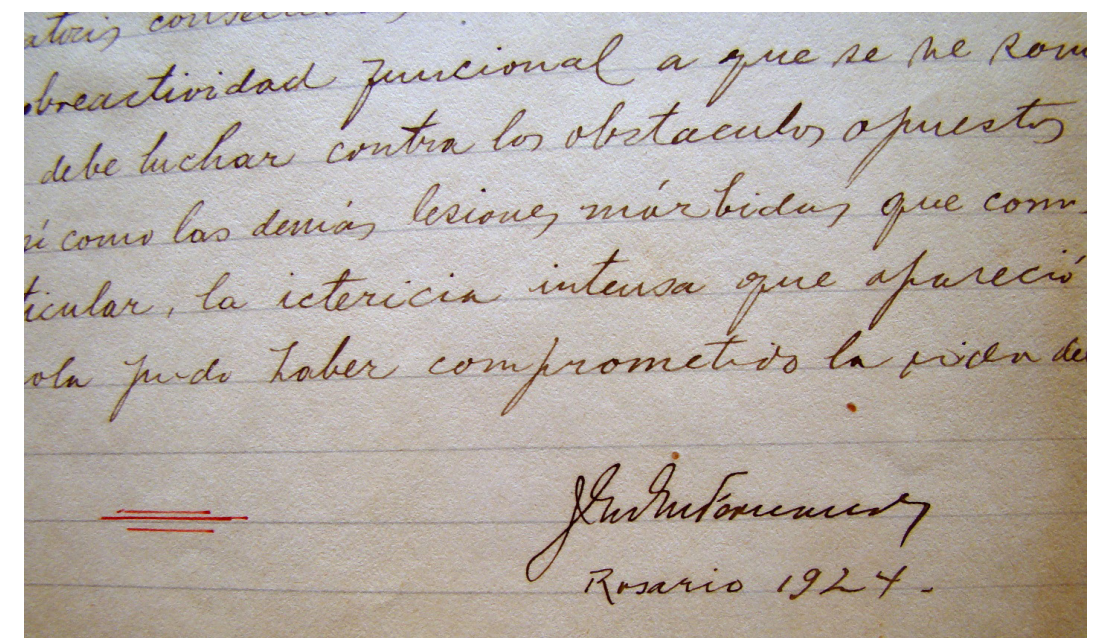

Figura 4: TE de José María Fernández.

Su trabajo especial, 364/1924 (Figura 4) está escrito a mano, una letra perfecta, casi un dibujo. Realizó la autopsia de un joven de 19 años que presentaba una neoplasia mesenquimática (interpretada como rabdomiosarcoma) a nivel del cuello y que en el transcurso de 6 meses diseminó en casi todos los órganos.

¡Sería interminable mencionar todos los trabajos!

En esos años una mujer estudiando medicina no era algo habitual. Vaya nuestro reconocimiento a aquellas pioneras: Dra María Boljover de Uriarte TE 230/1924, recibida en 1930, Dra Clara Gordon TE 1024/1927 recibida en 1931. Lamentablemente de las Dras Adelina Adelma Gossweiler y Sara Bercoff (títulos obtenidos en 1930 y 1931 respectivamente) no se logró encontrar los trabajos en el archivo.

\section{Broche final: hasta siempre Maestro}

Ha sido este trabajo un viaje al pasado, un volver a épocas en que el tiempo se detenía, cada día, cada hora se vivía intensamente, quizá hacían propias, con otra connotación, las palabras del Fausto de Goethe:

Verweile doch! du bist so schön! (minuto eres bello detente). 
Maestro Ruiz: quienes integramos actualmente la Cátedra de Patología le brindamos nuestro agradecimiento por habernos dejado este legado:

\section{Los alumnos no sólo tienen que adquirir el conocimiento sino que fundamentalmente deben lograr comprender la importancia del correlato anatomoclínico: cimiento fundamental de la medicina.}

\section{Agradecimientos}

A la Dra Maria del Luján Corbo por el aporte brindado en la realización del presente trabajo.

Fuente de financiamiento: no presenta.

\section{Referencias Bibliográficas}

1. Berra H. Hospital del Centenario de Rosario. Facultad de Ciencias Médicas, Farmacia y Ramos Menores. Universidad Nacional del Litoral. Cronología. Raíces y Emociones 1997; 2: 7-17.

2. Berra H. Hospital del Centenario de Rosario. Facultad de Ciencias Médicas, Farmacia y Ramos Menores. Universidad Nacional del Litoral. Apuntes sobre sus orígenes. Raíces y Emociones 1997; 1:5-18

. Salinas J, Agudo Avila A. Universidad Nacional del Litoral. Plan de estudios de la Facultad de Ciencias Médicas. Farmacia y Ramos Menores. Rev Med Litoral 1921; 189-206

5. Alloatti O. Fernando Román Ruiz. De Telémaco Susini a Juan Pedro Picena. Raíces y Emociones 1997; 2: 18-31

6. Federico Pergola. Historia de la Medicina Argentina. Ed 1. CABA: EUDEBA; 2014

7. Breve historia de la Cátedra de Patología (ex Anatomía Patológica) de la Facultad de Medicina ( UBA) . Sanchez N, Gelpi R, Napoli J. Médicos y Medicinas en la Historia 2019; XIV (39): 3-13

8. Venturini A. Dra. Cecilia Grierson. Médicos y Medicinas en la Historia 2019; XIV (39): 41

9. Picena J. Un maestro de la Patologia Argentina el Prof. Fernardo R Ruiz. Bol. A.N de Medicina 1971; 49: 635—642

10. Ruiz F. Memoria del Instituto de Anatomía y Fisiología Patológicas de la Facultad de Medicina de Rosario. Rev Med Ros 1932; $22: 162.163$

11. Ruiz F. Contribución al estudio de los tumroes de testículo. Revista Médica del Litoral 1921; 1: 55—69

12. Ruiz F. Varias. Rev Med Ros 1932; 22 (7):570

13. Lando M. Au Revoir...Rev Med Litoral 1921; 1/2): 117-118

14. Berón de Astrada. Elogio de "el gordo Ruiz". Rev Med Litoral 1921; 1(2):119-120

15. Valenti J, Nocito A. Historia de la Cátedra de Anatomía y Fisiología Patológicas. www.patologiafcm.com.ar

16. Berra H. Francisca Montaut de Osuna: primera médica graduada en la Facultad de Medicina de Rosario. Rev Med.Ros 2012; 78:145-152

17. Berra H. La foto historica Rev. Méd. Rosario 2012; 78: 158.

18. Fundacion Facultad de Cs Médicas. José María Fernandez.https://www.fucimed.org/actividades—ampliada.php?id=241

19. Fernandez J. The early reaction induced by lepromin. Int J Lepr 1940; 8: 1-14

20. UNL https://www.unl.edu.ar/institucional/galeria—de-rectores/pr 